\title{
37 Lehrerinnen- und Lehrerbildung an Universitäten und Pädagogischen Hochschulen
}

\author{
Lena Hollenstein, Christian Brühwiler und Horst Biedermann
}

\begin{abstract}
Aus dem Vergleich der Lehrerinnen- und Lehrerbildungsstrukturen an Universitäten und Pädagogischen Hochschulen in Deutschland, Österreich und der Schweiz sowie der Diskussion des Forschungsstands zeigt sich, dass sich die beiden Hochschultypen annähern: Während die universitäre Lehrerinnen- und Lehrerbildung vermehrt Praxisbezüge integriert, verstärken die Pädagogischen Hochschulen Wissenschaft und Forschung.
\end{abstract}

\section{Beschreibung der Struktur}

Die Lehrerinnen- und Lehrerbildung ist in Deutschland, Österreich und der Schweiz auf tertiärer Stufe angesiedelt. In allen drei Ländern findet die Lehrerinnen- und Lehrerbildung an Universitäten und Pädagogischen Hochschulen statt, allerdings unterscheiden sie sich in ihren Modellen. Nachfolgend werden die Aspekte Organisationsstrukturen (vgl. 1.1), Zugangsvoraussetzungen (vgl. 1.2), Studienprogramme (vgl. 1.3) sowie Lehrberechtigung (vgl. 1.4) entlang der drei Länder dargestellt.

\subsection{Organisationsstrukturen}

In Deutschland findet die Lehrerinnen- und Lehrerbildung einheitlich an Hochschulen mit Promotions- und Habilitationsrecht statt und ist zweiphasig organisiert, worin zwei zentrale Unterscheidungsmerkmale gegenüber den beiden anderen betrachteten Ländern liegen. In der ersten Phase erfolgt das Studium an einer Universität bzw. Pädagogischen Hochschule, die zweite Phase umfasst den Vorbereitungsdienst bzw. das Referendariat an Studienseminaren ( $\rightarrow$ Kapitel 38). Aufgrund eines Ministerratsbeschlusses im Jahr 2003 benötigt es in Deutschland an Universitäten mit Studienangeboten in der Lehrerinnen- und Lehrerbildung entweder ein Lehrerbildungszentrum oder eine „School of Education“. Dabei handelt es sich um „zentrale, fachbereichsübergreifende Einrichtungen zur Koordination und Steuerung in der Lehrerausbildung “ (Weyand \& Schnabel-Schüle, 2010, S. 9). An Pädagogischen Hochschulen, wie sie in Baden-Württemberg zu finden sind, bedarf es keine solchen Einrichtungen, da die Pädagogischen Hochschulen explizit auf Studiengänge im Bildungsbereich - besonders der Lehrerinnen- und Lehrerbildung spezialisiert sind. Die Pädagogischen Hochschulen unterscheiden sich von den Universitäten somit ausschließlich durch die thematische Fokussierung des Studienangebots. An Universitäten mit Lehrerinnen- und Lehrerbildung können in Deutschland alle Lehramtstypen studiert werden (Primarstufe, Sekundarstufe I und II), wobei einzelne Hochschulstandorte Studienangebote nur für ausgewählte Lehramtstypen vorhalten. An den Pädagogischen Hochschulen werden die Lehrämter der Primarstufe- und Sekundarstufe I angeboten, nicht aber das Gymnasiallehramt (Döbert, von Kopp \& Weishaupt, 2014). 
In Österreich stellt es eine lange Tradition dar, dass die Lehrerinnen- und Lehrerbildung sowohl an Pädagogischen Hochschulen (ehemals Pädagogische Akademien) als auch an Universitäten stattfindet. Die Pädagogischen Hochschulen waren zuständig für die Ausbildung von Lehrpersonen der Primarstufe und der Sekundarstufe I, die Universitäten für die Ausbildung zur Gymnasiallehrperson (Schratz, 2012; Gräsel, 2019). Über einen langen Zeitraum unterschieden sich die Pädagogischen Hochschulen von den Universitäten dadurch, dass deren Ausbildung kürzer war und mehr Praxisanteile enthielt. Demgegenüber waren die fachwissenschaftlichen Anteile im Vergleich zu den Universitäten geringer. Mit der 2013 initiierten Reform PädagogInnenbildung NEU wird unter anderem das Ziel verfolgt, die Studienprogramme von Pädagogischen Hochschulen und Universitäten anzugleichen, wobei insbesondere die Fachwissenschaften an den Pädagogischen Hochschulen gestärkt werden sollen. Die neuen Programme starteten 2015 flächendeckend für die Primarstufe und 2016 für die Sekundarstufe (Eder, 2014; Braunsteiner \& Spiel, 2019). Auch heute wird das Lehramt für die Primarstufe ausschließlich von den Pädagogischen Hochschulen angeboten (BGB, 2013). Für die Ausbildung von Lehrpersonen der Sekundarstufe I müssen Pädagogische Hochschulen mit Universitäten kooperieren, wohingegen Universitäten diesen Studiengang ohne Kooperation mit Pädagogischen Hochschulen anbieten können (BGB, 2013; Drahmann, Rothland, König \& Pflanzl, 2016). Mit der Reform wird neu auch eine einjährige Induktionsphase verlangt, in der die regulär unterrichtenden Lehrpersonen in ihrem ersten Berufsjahr von Mentorinnen bzw. Mentoren an den jeweiligen Schulen begleitet werden. Die Mentorinnen und Mentoren werden für die Begleitung der Berufseinsteigenden an den Pädagogischen Hochschulen ausgebildet. Die Induktionsphase kann wahlweise nach dem Bachelorabschluss, der zu einer Unterrichtstätigkeit von maximal fünf Jahren berechtigt, nach dem Masterabschluss oder auch parallel zum Masterstudium absolviert werden (Frank \& Harisch-Tresch, 2015; Krainz-Dürr, 2015).

In der Schweiz wurde die Tertiarisierung der Lehrerinnen- und Lehrerbildung erst ab den 1990er Jahren eingehend diskutiert. In den meisten Kantonen wurde zwischen 2001 und 2004 der Reformprozess mit der Schaffung neuer Studiengänge an Pädagogischen Hochschulen (vorläufig) abgeschlossen (Oser \& Oelkers, 2001; Lehmann, Criblez, Guldimann, Fuchs \& Périsset Bagnoud, 2007). Im Vergleich zu Deutschland und Österreich erfolgte die Tertiarisierung somit spät. Seit 2015 ist das Hochschulförderungs- und-koordinationsgesetz (HFKG) in Kraft, das die drei Hochschultypen (Universität/Eidgenössische Technische Hochschule, Fachhochschule und Pädagogische Hochschule) als gleichwertig, aber andersartig anerkennt (HFKG, 2020). Dennoch sind die Pädagogischen Hochschulen vereinzelt organisatorisch in Fachhochschulen eingegliedert (z. B. Pädagogische Hochschule der Fachhochschule Nordwestschweiz). Die Lehrerinnen- und Lehrerbildung erfolgt einphasig, d.h. die Praxisphasen sind in das Studium integriert. Die Besonderheit des Schweizer Schulsystems ist - im Vergleich zu Deutschland und Österreich, dass auch Lehrpersonen des Kindergartens an Pädagogischen Hochschulen ausgebildet werden. Der Kindergarten zählt hier zur obligatorischen Schule. Die angehenden Lehrpersonen erhalten mit ihrem Bachelor- (Kindergarten- und Primarstufe) bzw. Masterdiplom (Sekundarstufe I) ihre Lehrbefähigung. Je nach Kanton absolvieren die Lehrpersonen während ihres ersten Berufsjahres eine (in der Mehrzahl der Kantone obligatorische) Berufseinführung, die von einer Pädagogischen Hochschule, der kantonalen Bildungsverwaltung oder den lokalen Schulleitungen verantwortet wird (Picard \& Ria, 2011; EDK, 2020b). Die Ausbildung von Kindergartenund Primarlehrpersonen sowie Lehrpersonen der Sekundarstufe I findet, analog zu Österreich, mehrheitlich an Pädagogischen Hochschulen statt, vereinzelt jedoch auch an Universitäten (wobei sich dies primär auf die französischsprachige Schweiz bezieht). Zudem werden von einzelnen Pädagogischen Hochschulen Studienprogramme zur Lehrperson in beruflicher Bildung und in schulischer Heilpädagogik durchgeführt. Die Universitäten sind demgegenüber mehrheitlich 
für die Studiengänge des Gymnasiallehramts zuständig. Wenn Pädagogische Hochschulen diese Angebote unterbreiten, dann in der Regel in Kooperation mit Universitäten. Einzelne Universitäten bieten Studiengänge für das Lehramt der Sekundarstufe I an (EDK, 2020a).

\subsection{Zugangsvoraussetzungen}

Die Lehrerinnen- und Lehrerbildung in Deutschland und Österreich setzt als Zugangsvoraussetzung eine allgemeine Hochschulreife voraus (für detaillierte Informationen: $\rightarrow$ Kapitel 43). Zulassungsbeschränkungen existieren in Deutschland an stark nachgefragten Standorten durch die Abiturnote. Zudem können Eignungsabklärungen stattfinden, beispielsweise durch (Selbst-) Reflexionsprozesse oder zu absolvierende Praxiserfahrung vor Beginn des Studiums (Eignungsund/oder Orientierungspraktikum) (Nieskens, 2016). In Österreich müssen alle Lehramtskandidatinnen und -kandidaten ein Aufnahme- und Eignungsfeststellungsverfahren durchlaufen, auf dessen Grundlage die studien- und berufsbezogene Eignung überprüft wird (BMBWF, 2020).

In der Schweiz gilt ebenfalls grundsätzlich die allgemeine Hochschulreife als Zulassungsvoraussetzung. Aufnahmebeschränkungen basierend auf Noten oder Eignungsabklärungen gibt es keine. Insbesondere für das Studium zur Kindergarten- und Primarlehrperson können auf verschiedenen Wegen äquivalente Leistungen erbracht werden, die zu einer Zugangsberechtigung führen (z. B. Fachmaturität Pädagogik, Maturität für Erwachsene auf dem zweiten Bildungsweg; vgl. Criblez, 2007; SKBF, 2018). Aus diesem Grund können die Studierenden in ihren Leistungsvoraussetzungen und ihrem Vorwissen im Vergleich zu Deutschland und Österreich deutlich heterogener sein (Biedermann, Tettenborn, Oser, Steinmann \& Bach, 2015).

\subsection{Studienprogramme: Organisation, Inhalte und Umfang}

In der Regel schließen angehende Lehrpersonen in Deutschland die erste Phase der Lehrerinnen- und Lehrerbildung - unabhängig von der Zielstufe - mit einem Master (B.A. 180 ECTS und M.A. 120 ECTS) ab (KMK, 2019). In der deutlichen Mehrheit der Bundesländer ist mittlerweile während des Masters ein Langzeitpraktikum von einem Semester implementiert (Weyland \& Wittmann, 2015; Ulrich et al., 2020). Dies hat zum Ziel, trotz der zweiphasigen Lehrerbildung mit einer grundsätzlichen Aufteilung von Wissenschaft und Praxis bereits während des Studiums die unmittelbare Vernetzung von Theorie und Praxis zu ermöglichen (Gröschner, 2019).

In Österreich schließen seit der Reform PädagogInnenbildung NEU angehende Lehrpersonen für die Primarstufe mit einem Master of Education ab. Insgesamt sind 300 ECTS (B. A. 240 ECTS und M.A. mindestens 60 ECTS) in einem Umfang von mindestens 10 Semestern zu absolvieren. Das Studium zur Sekundarlehrperson umfasst mindestens 11 Semester (M.A. mindestens 90 ECTS) (BMBWF, 2020). Berufspraktische Erfahrungen sammeln die Studierenden mit Praktika (insgesamt 50 ECTS) sowie didaktischen Reflexionsveranstaltungen an den Pädagogischen Hochschulen (Hascher \& Winkler, 2017). Seit der Umsetzung der Reform ab 2015 ist das Verhältnis von wissenschaftlichen und praktischen Inhalten nicht mehr von der Zielstufe abhängig (Gräsel, 2019).

In der Schweiz schließt das Studium zur Kindergarten- und Primarstufenlehrperson mit einem sechssemestrigen Bachelor of Arts ab. Von den 180 ECTS sind 36-54 ECTS für die berufspraktische Ausbildung einzusetzen (EDK, 2020c). Lehrpersonen der Sekundarstufe I schließen nach fünf Jahren Studium mit einem Master of Arts ab. Nach dem Bachelor müssen 120 ECTS (davon mindestens 48 ECTS für berufspraktische Studien) absolviert werden. Das Studium zur Lehrperson auf der Sekundarstufe II umfasst ein fünfähriges fachbezogenes Universitätsstudium (300 ECTS) sowie ein Jahr der pädagogisch-didaktischen Vertiefung ebenfalls an der Universität 
oder an einer Pädagogischen Hochschule (60 ECTS). Dabei sind pro Unterrichtsfach (in der Regel zwei Fächer) 15 ECTS für Praktika vorgesehen, die restlichen ECTS für pädagogischpsychologische sowie fachdidaktische Inhalte (Hascher \& Winkler, 2017; SKBF, 2018).

\subsection{Lehrberechtigung}

In Deutschland erhalten Lehrpersonen aller Zielstufen ihre Lehrberechtigung für zwei bis vier Fächer nach erfolgreicher Beendigung des Vorbereitungsdiensts (18-24 Monate; detaillierte Ausführungen: $\rightarrow$ Kapitel 38).

In Österreich und der Schweiz erhalten Lehrpersonen der Primarstufe - deren Ausbildung zur großen Mehrheit an Pädagogischen Hochschulen stattfindet - eine Lehrberechtigung für alle Fächer (Generalistinnen- und Generalistenausbildung). Lehrpersonen der Sekundarstufe erhalten, analog zu Deutschland, eine Lehrbefähigung für zwei bis vier Fächer (Biedermann et al., 2015; BMBWF, 2020).

\section{Forschungsstand}

Die Frage, ob die Ansiedelung der Lehrerinnen- und Lehrerbildung an Universitäten oder Pädagogischen Hochschulen Unterschiede in der Entwicklung der professionellen Kompetenz von angehenden Lehrpersonen erzeugt, ist äußerst komplex, weshalb bisher kaum empirische Forschung dazu vorliegt (Hascher, 2014). Ebenso gibt es keine empirisch belastbaren Studien, die sich explizit damit befassen, inwiefern sich die Phasenstruktur (einphasig vs. zweiphasig) auf den Ausbildungserfolg auswirkt (vgl. Hascher \& Winkler, 2017).

Mit Blick auf die Eingangsvoraussetzungen konnten Drahmann et al. (2016) anhand einer Studie zur Entwicklung von berufsspezifischer Motivation und pädagogischem Wissen in der Lehrerausbildung (EMW) für die Stichprobe aus Österreich aufzeigen, dass Studierende der Pädagogischen Hochschulen höhere altruistische bzw. soziale Berufswahlmotive aufweisen als die Studierenden an Universitäten. Inwieweit dies tatsächlich auf eine bewusste Entscheidung für eine der Institutionen oder auf den Studiengang zurückzuführen ist, kann jedoch nicht eindeutig beantwortet werden. Zum Zeitpunkt der Erhebung war in Österreich die Entscheidung für einen Studiengang gleichzeitig auch die Entscheidung für eine Institution.

In Studien zur Wirksamkeit der Lehrerinnen- und Lehrerbildung lassen sich gewisse Hinweise auf mögliche system- oder institutionell bedingte Unterschiede finden, wobei dabei unklar bleibt, inwieweit dafür Spezifika der Phasierung der Lehrerinnen- und Lehrerbildung oder institutionelle Merkmale eine Rolle spielen (Hascher \& Winkler, 2017). Generell lässt sich festhalten, dass empirische Untersuchungen zur Lehrerinnen- und Lehrerbildung lange vernachlässigt wurden und sich erst vor rund zwei Dekaden intensivierten - zunächst national und mittels Selbsteinschätzungen (z. B. in der Schweiz durch Oser \& Oelkers, 2001), erst später auch international und mittels standardisierter Leistungstests (z. B. TEDS-M: Tatto et al., 2012). Meist messen diese Studien die Entwicklung von Kompetenzdimensionen wie das fachspezifische und fachdidaktische Wissen, epistemologische Überzeugungen und motivationale Selbstregulation während des Studiums (z.B. LEK: König, 2010) oder am Ende des Studiums (z. B. MT21: Blömeke, Kaiser \& Lehmann, 2008; TEDS-M: Tatto et al., 2012).

Die wohl meist beachtete international vergleichende Lehrerbildungsstudie ist TEDS-M (Teacher Education and Development Study in Mathematics; Tatto et al., 2012), die Vergleiche zur Ausbildung von Mathematiklehrpersonen in Deutschland und der Deutschschweiz ermöglicht. In der Schweiz beschränkte sich die Studie auf die Deutschschweiz, Österreich hat sich an TEDS-M nicht beteiligt. Aus TEDS-M geht hervor, dass angehende Lehrpersonen mit einer 
Lehrbefähigung von der 1. bis zur 10. Klasse (Grund-, Haupt- oder Realschule) in Deutschland unter Berücksichtigung des mathematischen und mathematikdidaktischen Wissens im internationalen Durchschnitt liegen. Angehende Lehrpersonen mit einer Lehrbefähigung von der 5. bis zur 13. Klasse (Gymnasium) zeigen im internationalen Vergleich deutlich höhere Mittelwerte (Blömeke, Kaiser \& Lehmann, 2010a, 2010b). In der Deutschschweiz erzielen künftige Lehrpersonen sowohl in Mathematik als auch in Mathematikdidaktik Leistungen über dem internationalen Mittelwert (Oser, Biedermann, Brühwiler \& Steinmann, 2015). Bezüglich des Mathematikwissens ist dies angesichts der strukturellen Besonderheit der schweizerischen Primarlehrpersonenausbildung bemerkenswert, denn es bestehen während des Studiums kaum fachwissenschaftliche Lerngelegenheiten in der Mathematik. Dementsprechend sind keine signifikanten Unterschiede im Mathematikwissen zwischen Studienbeginn und Studienabschluss festzustellen, was auch auf die große Bedeutung der Vorbildung hinweist (Brühwiler, Ramseier \& Steinmann, 2015). Auffallend bei der Betrachtung der Ergebnisse aus TEDS-M sind die großen Leistungsunterschiede innerhalb Deutschlands in Abhängigkeit des Studiengangs (Blömeke et al., 2010a; 2010b). In der Deutschschweiz fällt die Varianz der Studienleistungen zwar geringer aus als in Deutschland (Tatto et al., 2012), die Unterschiede zwischen den einzelnen Pädagogischen Hochschulen sind dennoch erheblich (Brühwiler et al., 2015). Dies könnte damit zusammenhängen, dass in der Schweiz kein einheitliches Ausbildungscurriculum gegeben ist (Krattenmacher, Brühwiler, Oser \& Biedermann, 2010), während in Deutschland die KMKStandards schon früh verabschiedet wurden (KMK, 2004).

Aus diesen Ergebnissen lässt sich schließen, dass die Varianz in den Leistungsergebnissen wohl weniger auf die unterschiedliche Phasigkeit noch auf den Hochschultyp (Universität oder Pädagogische Hochschule) zurückzuführen ist als vielmehr auf die unterschiedlichen Studiengänge oder institutionsspezifischen Rahmenbedingungen. Hierzu halten Darling-Hammond und Oakes (2019) fest, dass sich wirksame Lehrerinnen- und Lehrerbildung insbesondere durch geringe strukturelle und inhaltliche Fragmentierungen auszeichnet. Nach White und Forgasz (2016) stellt sich die Frage nach einer engen Verbindung von theoretischen und praktischen Ausbildungsinhalten, wobei gerade in den berufspraktischen Studienteilen eine enge und professionelle Begleitung der Studierenden von besonderer Bedeutung ist (Schubarth, 2011; Jones \& Ryan, 2014; Hascher \& Winkler, 2017; Mayer et al., 2017; Grossman, 2018; Zeichner, 2018). Dies stellt sowohl in der einphasigen als auch in der zweiphasigen Lehrerinnen- und Lehrerbildung an Universitäten und Pädagogischen Hochschulen eine bisher unzureichend gelöste Herausforderung dar. Inwieweit die Verzahnung von Theorie und Praxis an Universitäten oder Pädagogischen Hochschulen besser gelingt, kann basierend auf den bisherigen Studien zur Wirksamkeit von Praktika während des Studiums nicht gesagt werden (Bach, 2014; Ulrich \& Gröschner, 2020). Umsetzungsbeispiele lassen sich im deutschsprachigen Raum sowohl an Universitäten (Götz, Schumann, Begemann \& Crivellari, 2017) als auch an Pädagogischen Hochschulen finden, in der Schweiz z. B. im Rahmen von Projekten zur Stärkung des doppelten Kompetenzprofils (swissuniversities, 2020).

\section{Diskussion}

Die Ausführungen in Abschnitt 1 machen deutlich, dass sich Universitäten und Pädagogische Hochschulen vor allem in Bezug auf die dort zu studierenden Lehramtstypen und in der Schweiz auch in Bezug auf die Zulassungsvoraussetzungen unterscheiden. Ein bedeutsamer struktureller Unterschied zwischen den Ländern liegt darin, dass in Deutschland die Lehrerinnen- und Lehrerbildung ausschließlich an Hochschulen mit Promotions- und Habilitationsrecht angesiedelt 
ist. In Österreich und in der Schweiz findet die Lehrerinnen- und Lehrerbildung hingegen zu großen Teilen an Pädagogischen Hochschulen statt, die nicht über ein eigenes Promotionsrecht verfügen. Nichtsdestotrotz haben auch hier die Pädagogischen Hochschulen einen (berufsfeldbezogenen) Forschungsauftrag zu erfüllen (vgl. A: Hochschulgesetz, 2005, CH: Hochschulförderungs- und koordinationsgesetz, 2011). Der Forschungsfokus der Pädagogischen Hochschulen liegt auf professionsorientierter Forschung, d. h. die Forschung soll einen Beitrag leisten zur Weiterentwicklung der professionellen Wissensbasis des Lehrberufs (Forneck, 2015) und der Bildung. In Österreich und in der Schweiz führen Reformen und Gesetze in den letzten Jahren in Richtung einer Annäherung von Pädagogischen Hochschulen und Universitäten (Österreich: PädagogInnenbildung NEU, BGB, 2013; Schweiz: Hochschulförderungs- und koordinationsgesetz, HFKG, 2020). In Deutschland wird mit der Qualitätsoffensive Lehrerbildung die Verbesserung der Qualität der Lehrerinnen- und Lehrerbildung angestrebt (BMBF, 2016).

In Bezug auf die Studienprogramme, die Lehrbefähigung und den Berufseinstieg werden Unterschiede zwischen den Ländern, aber kaum zwischen den Institutionen ersichtlich. Wie die bisherigen Ausführungen deutlich machen, ist die Ausbildungsdauer je nach Land und Zielstufe sehr unterschiedlich (vgl. 1). Somit variiert auch der Praxisanteil während des Studiums bedeutsam.

In Anbetracht des oben aufgeführten Forschungsstands liegt keine empirisch abgesicherte Evidenz vor, welcher Hochschultypus und welches Lehrerbildungsmodell angehende Lehrpersonen am besten auf den Berufseinstieg vorbereitet. Mit der inzwischen überall vollzogenen Verortung der Lehrerinnen- und Lehrerbildung auf Tertiärstufe wird einem Mangel an Wissenschaftlichkeit entgegengewirkt. Die unmittelbare Vernetzung von wissenschaftlichen Inhalten und praktischen Erfahrungen ist weiter ausbaufähig (z. B. Zweigliedrigkeit in Deutschland) (Hascher \& Winkler, 2017; Gray \& Ramberg, 2019). In Anlehnung an das Kompetenzmodell von Blömeke, Gustafsson und Shavelson (2015) benötigt es sowohl theoretisches Wissen (Disposition) über einen gelingenden Unterricht und erfolgreiche Lernprozesse bei Schülerinnen und Schülern als auch praktisches Wissen (situationsspezifische Fähigkeiten), um das gelernte Wissen erfolgreich in der Unterrichtspraxis einbringen zu können (Performanz). Folglich sind die vermehrt aufkommenden Bestrebungen, Wissenschaft und Praxis näher zusammenzubringen, positiv zu bewerten (z. B. Shavelson, 2020), zugleich aber nicht trivial ( $\rightarrow$ Kapitel 15; 31). Angehende Lehrpersonen benötigen für die erfolgreiche Bewältigung ihrer beruflichen Anforderungen während ihrer Qualifikation Lerngelegenheiten, die eine nachhaltige Vernetzung von Wissenschaft und Praxis fördern (Jones \& Ryan, 2014; Hascher \& Winkler, 2017). Dies gelingt momentan im deutschsprachigen Raum (noch) nicht zufriedenstellend (Neuweg, 2016). Andere Länder scheinen diesbezüglich einen Schritt weiter zu sein. So werden beispielsweise in Finnland flächendeckend alle Praktika während des Studiums in so genannten ,Teacher Training Schools' absolviert, womit eine enge Zusammenarbeit zwischen Studierenden, Mentorinnen und Mentoren sowie Praktikumslehrpersonen besteht (Sahlberg, 2015). In Norwegen zeigt sich ein ähnliches Modell an der Universität Trondheim, das mittels Universitätsschulen ebenso eine systematische und dauerhafte Verknüpfung von Praxisschulen und Hochschule vorsieht, um die Qualität der Lehrerinnen- und Lehrerbildung positiv zu beeinflussen (Gray \& Ramberg, 2019).

Es gibt jedoch auch im deutschsprachigen Raum Bestrebungen, die darauf hinweisen, dass zukünftig die Vernetzung von Wissenschaft und Praxis vermehrt ins Zentrum rückt. Erste Forschungsprojekte haben zum Ziel, hybride Räume (Fraefel, 2018) in der Lehrerinnen- und Lehrerausbildung zu schaffen, die die Vernetzung von Wissenschaft und Praxis fokussieren (Götz et al., 2017; swissuniversities, 2020). Des Weiteren wurde in Deutschland ein Clearing House Unterricht aufgebaut, das als Schnittstelle zwischen Bildungsforschung und professioneller Praxis den Wissenstransfer unterstützt (Seidel et al., 2017). 
Zusammenfassend kann somit festgehalten werden, dass es bislang noch keine verlässlichen Daten über die Wirksamkeit der Lehrerinnen- und Lehrerbildung an Universitäten und/ oder Pädagogischen Hochschulen gibt, weshalb ein darauf ausgerichteter Vergleich nicht möglich ist. Um solche Fragen zu untersuchen, sind vermehrt international vergleichende Studien durchzuführen, die die unterschiedlichen Ausbildungsbedingungen systematisch erfassen und mit Kriterien des Ausbildungserfolgs in Verbindung bringen. Es zeigt sich jedoch, dass sich die beiden institutionellen Wege der Lehrerinnen- und Lehrerbildung immer stärker annähern: während die universitäre Lehrerinnen- und Lehrerbildung vermehrt Praxisbezüge in die Studienprogramme integriert, verstärken die Pädagogischen Hochschulen die Wissenschaftsund Forschungsbemühungen. Auch wenn der Prozess der Professionalisierung des Lehrerinnen- und Lehrberufs bis heute nicht abgeschlossen ist, so gehen die Entwicklungen in den letzten Jahren ungeachtet der institutionellen Verortung der Grundbildung zunehmend in diese Richtung.

\section{Empfehlungen zur vertiefenden Lektüre}

Bosse, D., Criblez, L., \& Hascher, T. (Hrsg.) (2012). Reform der Lehrerbildung in Deutschland, Österreich und der Schweiz I: Analysen, Perspektiven und Forschung. Immenhausen: Prolog-Verlag.

Braunsteiner, M.-L., \& Spiel, C. (Hrsg.) (2019). PädagogInnenbildung. Festschrift für Andreas Schnider. Heiligenkreuz: Be\&Be-Verlag.

Hascher, T., \& Winkler, A. (2017). Analyse einphasiger Modelle der Lehrer_innenbildung in verschiedenen Ländern. Frankfurt am Main: Gewerkschaft Erziehung und Wissenschaft.

Loughran, J., \& Hamilton, M. L. (Eds.) (2016). International Handbook of Teacher Education (Vol. $1 \&$ 2). Singapore: Springer.

Oser, F., Biedermann, H., Brühwiler, C., \& Steinmann, S. (Hrsg.) (2015). Zum Start bereit? Kritische Ergebnisse aus TEDS-M zur schweizerischen Lehrerbildung im internationalen Vergleich. Opladen: Barbara Budrich.

\section{Literatur}

Bach, A. (2014). Kompetenzentwicklung im Schulpraktikum. Ausmaß und zeitliche Stabilität von Lerneffekten hochschulischer Praxisphasen. Münster: Waxmann.

BGB [Bundesgesetzblatt] (2013). 124. Bundesgesetz: Bundesrahmengesetz zur Einführung einer neuen Ausbildung für Pädagoginnen und Pädagogen. https://www.parlament.gv.at/PAKT/VHG/XXIV/I/I_02348/ [21.02.2020].

Biedermann, H., Tettenborn, A., Oser, F., Steinmann, S., \& Bach, A. (2015). Die Lehrerbildungssysteme in der Deutschschweiz und in Deutschland. In F. Oser, H. Biedermann, C. Brühwiler \& S. Steinmann (Hrsg.), Zum Start bereit? Vertiefende Ergebnisse aus TEDS-M zur schweizerischen Lehrerbildung im internationalen und nationalen Vergleich (S. 47-72). Opladen: Budrich.

Blömeke, S., Gustafsson, J.-E., \& Shavelson, R. J. (2015). Beyond dichotomies competence viewed as a conitnuum. Zeitschrift für Psychologie, 223(1), 3-13.

Blömeke, S., Kaiser, G., \& Lehmann, R. (Hrsg.) (2008). Professionnelle Kompetenz angehender Lehrerinnen und Lehrer: Wissen, Überzeugungen und Lerngelegenheiten deutscher Mathematikstudierender und -referendare. Erste Ergebnisse zur Wirksamkeit der Lehrerausbildung. Münster: Waxmann.

Blömeke, S., Kaiser, G., \& Lehmann, R. (Hrsg.) (2010a). TEDS-M 2008. Professionelle Kompetenz und Lerngelegenheiten angehender Mathematiklehrkräfte für die Sekundarstufe I im internationalen Vergleich. Münster: Waxmann.

Blömeke, S., Kaiser, G., \& Lehmann, R. (Hrsg.) (2010b). TEDS-M 2008. Professionelle Kompetenz und Lerngelegenheiten angehender Primarstufenlehrkräfte im internationalen Vergleich. Münster: Waxmann.

BMBF [Bundesministerium für Bildung und Forschung] (Hrsg.) (2016). Neue Wege in der Lehrerbildung. Die Qualitätsoffensive Lehrerbildung. Berlin: BMBF. 
BMBWF [Bundesministerium Bildung, Wissenschaft und Forschung] (Hrsg.) (2020). Allgemeinbildung - Lehramtsstudien an den Pädagogischen Hochschulen - Bereich Primarstufe und Sekundarstufe. https://www.bmbwf.gv.at/Themen/ schule/fpp/ausb/ab.html [08.04.2020].

Braunsteiner, M.-L., \& Spiel, C. (2019). PädagogInnenbildung. Festschrift für Andreas Schnider. Heiligenkreuz: Be\&BeVerlag.

Brühwiler, C., Ramseier, E., \& Steinmann, S. (2015). Vorbildung oder Ausbildung? Zum Erwerb mathematischen und mathematikdidaktischen Wissens in der Lehrerausbildung. Beiträge zur Lehrerinnen- und Lehrerbildung, 33(1), $22-45$.

Criblez, L. (2007). Lehrerinnen-und Lehrerbildung in der Schweiz. Ausgewählte Ergebnisse aus der Bildungsberichterstattung. Beiträge zur Lehrerinnen- und Lehrerbildung, 25(3), 377-391.

Darling-Hammond, L., \& Oakes, J. (2019). Preparing Teachers for deeper learning. Cambridge: Harvard Education Press.

Döbert, H., von Kopp, B., \& Weishaupt, H. (2014). Innovative Ansätze der Lehrerbildung im Ausland und Anregungen für die deutsche Diskussion. In W. Bos, M. Krüger-Potratz, J. Henze, S. Hornberg, B. von Kopp, H.-G. Kotthoff \& P. J. Weber (Hrsg.), Innovative Ansätze in der Lehrerbildung im Ausland (S. 219-244). Münster: Waxmann.

Drahmann, M., Rothland, M., König, J., \& Pflanzl, B. (2016). Lehramtsstudierende an Pädagogischen Hochschulen und Universitäten - Zur Bedeutung motivationaler und kognitiver Eingangsvoraussetzungen für die Wahl der lehrerbildenden Institution und des Lehramtstypus. Lehrerbildung auf dem Prüfstand, 9(2), 227-252.

Eder, F. (2014). Die Ausbildung von Lehrerinnen und Lehrern in Österreich. In H. Döbert, B. von Kopp \& H. Weishaupt (Hrsg.), Innovative Ansätze der Lehrerbildung im Ausland (S. 149-165). Münster: Waxmann.

EDK [Schweizerische Konferenz der kantonalen Erziehungsdirektoren] (Hrsg.) (2020a). Institutionen der Lehreinnenund Lehrerbildung. http://www.edk.ch/dyn/13881.php [10.03.2020].

EDK [Schweizerische Konferenz der kantonalen Erziehungsdirektoren] (Hrsg.) (2020b). Lehrpersonen: Berufseinführung. http://www.edk.ch/dyn/30350.php [10.03.2020].

EDK [Schweizerische Konferenz der kantonalen Erziehungsdirektoren] (Hrsg.) (2020c). Reglement über die Anerkennung von Lehrdiplomen für den Unterricht auf der Primarstufe, der Sekundarstufe I und an Maturitätsschulen. https:// edudoc.educa.ch/static/web/arbeiten/diplanerk/anerkennung_lehrdiplome_regl_d.pdf [10.04.2020].

Forneck, H. J. (2015). Doppelter Bezug von pädagogischer Theorie und Praxis als zentrales Professionalisierungsziel. Beiträge zur Lehrerinnen- und Lehrerbildung, 33(3), 345-355.

Fraefel, U. (2018). Hybride Räume an der Schnittstelle von Hochschule und Schulfeld. Ein zukunftweisendes Konzept der Professionalisierung von Lehrpersonen. In L. Pilpaityte \& H.-S. Siller (Hrsg.). Schulpraktische Lehrerprofessionalisierung als Ort der Zusammenarbeit (S. 13-43). Wiesbaden: Springer.

Frank, E., \& Harisch-Tresch, M. (2015). Was ist neu an der Pädagog/innenbildung im Bereich der Primarstufe? PH_on.line, 9, 15-16.

Götz, T., Schumann, S., Begemann, M., \& Crivellari, F. (2017). Grenzen überwinden - Lernkulturen vernetzen: Die Binational School of Education der Universität Konstanz. journal für lehrerInnenbildung, 3, 39-43.

Gräsel, C. (2019). Die neue Pädagog*innenbildung in Österreich: Ein Kommentar zu drei zentralen Merkmalen der Reform. In M.-L. Braunsteiner \& C. Spiel (Hrsg.), PädagogInnenbildung. Festschrift für Andreas Schnider (S. 161-170). Heiligenkreuz: Be\&Be-Verlag.

Gray, P., \& Ramberg, P. J. (2019). Servants of the School: Building Teacher Education in Mid-Norway, 1998-2018. Trondheim: NTNU.

Gröschner, A. (2019). Zum Verhältnis der „Theorie“ zur „Praxis“: Anknüpfungen an John Dewey sowie Perspektiven zur Gegenwart und Zukunft der praxisbezogenen Lehrerausbildung. In J. Kosinar, A. Gröschner \& U. Weyland (Hrsg.), Langzeitpraktika als Lernräume - Historische Bezüge, Konzeptionen und Forschungsbefunde (S. 41-51). Münster: Waxmann.

Grossman, P. (2018). Teaching Core Practices in Teacher Education. Cambridge: Harvard Education Press.

Hascher, T. (2014). Forschung zur Wirksamkeit der Lehrerbildung. In E. Terhart, H. Bennewitz \& M. Rothland (Hrsg.), Handbuch der Forschung zum Lehrberuf (2. Aufl., S. 542-571). Münster: Waxmann.

Hascher, T., \& Winkler, A. (2017). Analyse einphasiger Modelle der Lehrer_innenbildung in verschiedenen Ländern. Frankfurt am Main: Gewerkschaft Erziehung und Wissenschaft.

HFKG [Hochschulförderungs- und koordinationsgesetz] (Hrsg.) (2020). Bundesgesetz über die Förderung der Hochschulen und die Koordination im schweizerischen Hochschulbereich. https://www.admin.ch/opc/de/classified-compilation/ 20070429/202001010000/414.20.pdf [10.04.2020].

Jones, M., \& Ryan, J. (2014). Successful teacher education: Partnerships, reflective practice and the place of technology. Amsterdam: Sense.

KMK [Kultusministerkonferenz] (Hrsg.) (2004). Standards für die Lehrerbildung: Bildungswissenschaften. Berlin: KMK. KMK [Kultusministerkonferenz] (Hrsg.) (2019). Sachstand in der Lehrerbildung. https://www.kmk.org/fileadmin/ Dateien/pdf/Bildung/AllgBildung/2019-11-05-Sachstand_LB-veroeff.pdf [08.04.2020]. 
König, J. (2010). Längsschnittliche Erhebung pädagogischer Kompetenzen von Lehramtsstudierenden (LEK). Theoretischer Rahmen, Fragestellungen, Untersuchungsanlage und erste Ergebnisse zu Lernvoraussetzungen von angehenden Lehrkräften. Lehrerbildung auf dem Prüfstand, 3(1), 56-83.

Krainz-Dürr, M. (2015). PädagogInnenildung NEU. PH_on.line, 9, 6-10.

Krattenmacher, S., Brühwiler, C., Oser, F., \& Biedermann, H. (2010). Was angehende Lehrpersonen in den Erziehungswissenschaften lernen sollten: Curriculumanalyse der erziehungswissenschaftlichen Ausbildung an den Deutschschweizer Lehrerausbildungsinstitutionen. Schweizerische Zeitschrift für Bildungswissenschaften, 32(1), 59-84.

Lehmann, L., Criblez, L., Guldimann, T., Fuchs, W., \& Périsset Bagnoud, D. (2007). Lehrerinnen- und Lehrerbildung in der Schweiz. Bericht im Rahmen der Bildungsberichterstattung 2006. Aarau: Schweizerische Koordinationsstelle für Bildungsforschung.

Mayer, D., Dixon, M., Kline, J., Kostogriz, A., Moss, J., Rowan, L., Walker-Gibbs, B., \& White, S. (2017). Studying the effeciveness of teacher education. Early Career Teachers in diverse settings. Singapore: Springer.

Neuweg, G. H. (2016). Praxis in der Lehrerinnen- und Lehrerbildung: Wozu, wie und wann? Rückfragen an eine lehrerbildungsdidaktische Einigungsformel. In J. Kosinar, S. Leineweber \& E. Schmid (Hrsg.), Professionalisierungsprozesse angehender Lehrpersonen in den berufspraktischen Studien (S. 31-46). Münster: Waxmann.

Nieskens, B. (2016). Eignungsabklärung und Zulassungssteuerung für den Lehrerberuf: Perspektiven, Instrumente und Erfahrungen. In A. Boeger (Hrsg.), Eignung für den Lehrerberuf(S. 155-179). Wiesbaden: Springer.

Oser, F., \& Oelkers, J. (Hrsg.) (2001). Die Wirksamkeit der Lehrerbildungssysteme. Von der Allrounderbildung zur Ausbildung professioneller Standards. Chur: Verlag Rüegger.

Oser, F., Biedermann, H., Brühwiler, C., \& Steinmann, S. (Hrsg.) (2015). Zum Start bereit? Kritische Ergebnisse aus TEDS-M zur schweizerischen Lehrerbildung im internationalen Vergleich. Opladen: Barbara Budrich.

Picard, P., \& Ria, L. (2011). Beginning teachers: a challenge for educational systems - CIDREE Yearbook 2011. Lyon: ENS de Lyon, Institut français de l'Éducation.

Sahlberg, P. (2015). Finnish lessons 2.0: What can the world learn from educational change in Finland? New York: Teachers College Press.

Schratz, M. (2012). Die Neuordnung der Lehrerbildung in Österreich. In D. Bosse, L. Criblez \& T. Hascher (Hrsg.), Reform der Lehrerbildung in Deutschland, Österreich und der Schweiz, Teil 1: Analysen, Perspektiven und Forschung (S. 93-108). Kassel: Prolog-Verlag.

Schubarth, W. (2011). Endlich Praxis! Forschungsbefunde zur Potenzen und Problemen der zweiten Phase der LehrerInnenbildung. journal für lehrerInnenbildung, 11(3), 31-40.

Seidel, T., Knogler, M., Mok, S., Hetmanek, A., Bauer, J., Vogel, F., \& Lankes, E. M. (2017). Forschung fördert (Lehrer) Bildung. Das Clearing House Unterricht. journal für lehrerInnenbildung, 17(3), 23-38.

Shavelson, R. J. (2020). Research on teaching and the education of teachers: Brokering the gap. Beiträge zur Lehrerinnen- und Lehrerbildung, 38(1), 37-53.

SKBF (2018). Swiss Education Report 2018. Aarau: Schweizerische Koordinationsstelle für Bildungsforschung.

swissuniversities (2020). P-11 Doppeltes Kompetenzprofil. https://www.swissuniversities.ch/themen/nachwuchs foerderung/p-11-doppeltes-kompetenzprofil [08.04.2020].

Tatto, M.T., Schwille, J., Senk, S. L., Ingvarson, L., Rowley, G., Peck, R., Bankov, K., Rodriguez, M., \& Reckase, M. (2012). Policy, practice, and readiness to teach primary and secondary mathematics in 17 countries. Findings from the IEA Teacher Education and Development Study in Mathematics (TEDS-M). Amsterdam: IEA.

Ulrich, I., \& Gröschner, A. (2020). Praxissemester im Lehramtsstudium in Deutschland: Wirkungen auf Studierende. Wiesbaden: Springer.

Ulrich, I., Klingebiel, F., Bartels, A., Staab, R., Scherer, S., \& Gröschner, A. (2020). Wie wirkt das Praxissemester im Lehramtsstudium auf Studierende? Ein systematischer Review. In I. Ulrich \& A. Gröschner (Hrsg.), Praxissemester im Lehramtsstudium in Deutschland: Wirkungen auf Studierende (S. 1-66). Wiesbaden: Springer.

Weyand, B., \& Schnabel-Schüle, H. (2010). Erhebung von Grunddaten zu Zentren für Lehrerbildung in Deutschland 2010. Trier: Stifterverband für die Deutsche Wissenschaft.

Weyland, U., \& Wittmann, E. (2015). Langzeitpraktika in der Lehrerausbildung in Deutschland - Stand und Perspektiven. journal für lehrerInnenbildung, 15(1), 8-21.

White, S., \& Forgasz, R. (2016). The practicum: The place of experience? In J. Loughran \& M. L. Hamilton (Eds.), International Handbook of teacher education (pp. 231-266). Singapore: Springer.

Zeichner, K. M. (2018). The struggle for the soul of teacher education. New York: Routledge. 